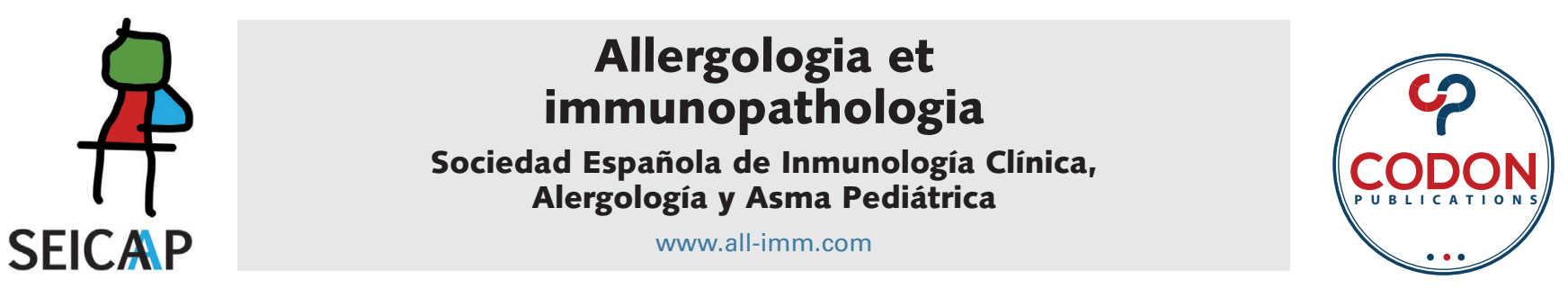

LETTER

OPEN ACCESS (C) (우)

\title{
Air quality and the COVID-19 outbreak in China
}

\author{
Wen-tao Gan ${ }^{a \neq}$, Meng-Yun Zhou ${ }^{c \neq}$, Yong-ran Cheng ${ }^{\mathrm{b}, c \neq}$, Lan Ye ${ }^{\mathrm{d}}$, Juan Chen ${ }^{\mathrm{a}}$, Ming-Wei \\ Wang $^{\text {a*}}$, Zhan-hui Feng ${ }^{\text {** }}$
}

\author{
${ }^{a}$ Affiliated Hospital of Hangzhou Normal University, Hangzhou, China. \\ ${ }^{b}$ Hangzhou Medical college, Hangzhou, China. \\ 'Department of Molecular \& Cellular Physiology, Shinshu University School of Medicine, Asahi, Matsumoto, Japan. \\ ${ }^{d}$ Basic medical college, Guizhou Medical University, Guizhou, China. \\ eDepartment of Neurology, Affiliated Hospital of Guizhou Medical University, Guiyang, China. \\ ₹Wen-tao Gan, Meng-Yun Zhou and Yong-ran Cheng contributed equally to this work
}

Received 25 July 2020; Accepted 8 August 2020

Available online: 2 January 2021

\section{KEYWORDS \\ Air quality; \\ COVID-19; \\ China}

We appreciate that Urrutia-Pereira M., et al. have reported on "COVID-19 and air pollution: A dangerous association." Research on the role of air pollution in the spread of SARS-CoV-2 is worth discussing. Air pollution refers to harmful substances that are carried in the air. When the quantity or proportion of the pollutants reaches or exceeds the environmental carrying capacity, they will change the normal state of the environment. ${ }^{2}$ With rapid economic development around the world, global pollution gradually increases. Ecological governance has become a crucial issue across the globe. ${ }^{3}$ Pollution control is also a highly debated issue between developing countries and developed countries; China too has an environmental pollution control problem. ${ }^{4}$

China began to pay attention to the question of pollution control and its prevention methods early on. The Chinese government has formulated many policies in this respect. However, these measures did not improve the environment. At the end of 2019, the COVID-19 epidemic broke in Wuhan, China. ${ }^{5}$ The Chinese government has taken a series of measures to control the outbreak. Wuhan was locked down on January 23, 2020, and people's travel was restricted.

We downloaded the daily air pollution parameters of 367 cities around China from the China Meteorological Administration website (http://data.cma.cn/). Records covered the periods between January 23, 2019 and March 23, 2019; November 22, 2019 and January 22, 2020; and January 23, 2020 and March 23, 2020. Pollution severity was divided into quartiles according to the air quality index (AQI). The AQI was calculated using the concentration of the following six pollutants: PM10, $\mathrm{PM}_{2.5}, \mathrm{CO}, \mathrm{NO}_{2}, \mathrm{SO}_{2}$, and $\mathrm{O}_{3}$. An AQI of 0-100 indicates no pollution, 101-150 indicates mild pollution, 151-200 indicates moderate pollution, and AQI > 200 indicates severe pollution (USEPA 2013, http://www.Gpo.gov/). We found a significant improvement in air quality across China as a result of the reduction

${ }^{*}$ Corresponding author: Ming-Wei Wang, MD., PhD, Affiliated Hospital of Hangzhou Normal University, Hangzhou, China. E-mail address: 495086736@qq.com; Zhan-hui Feng, MD., PhD. E-mail address: h9450203@126.com 


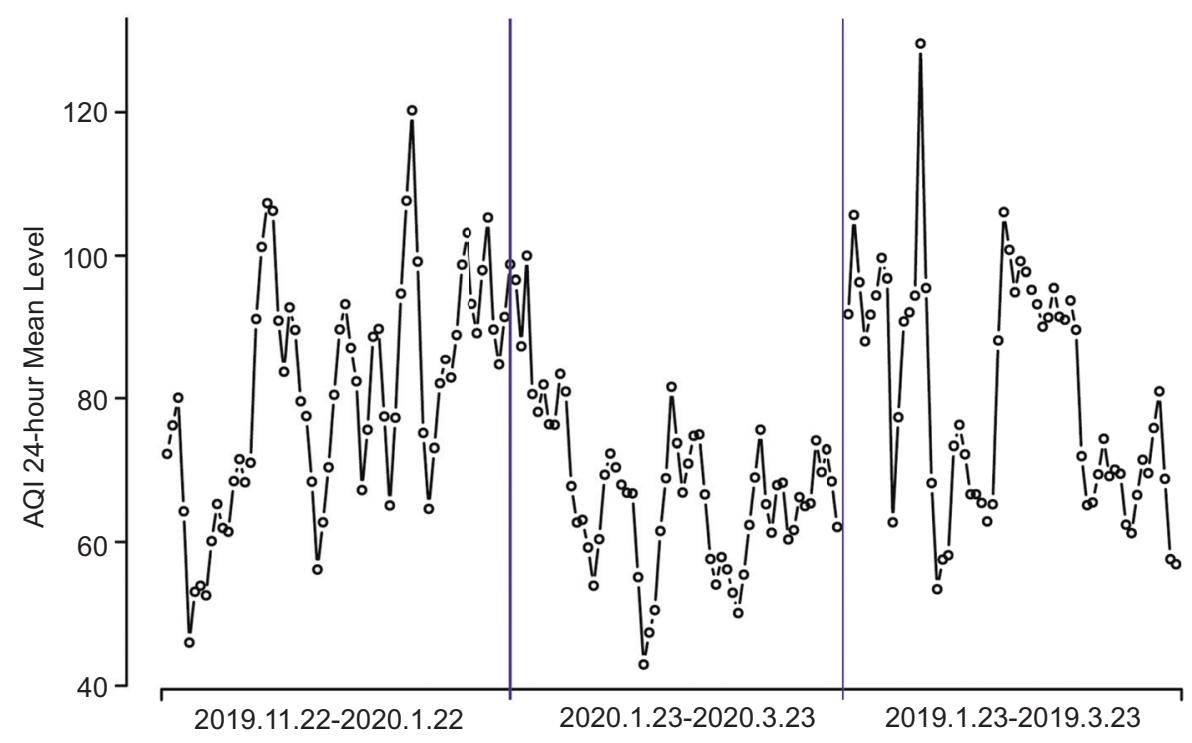

Figure 1 Air quality index comparison of three sections in 367 cities in China: November 22, 2019-January 22, 2020; January 23, 2020-March 23, 2020; and January 23, 2019-March 23, 2019.

A

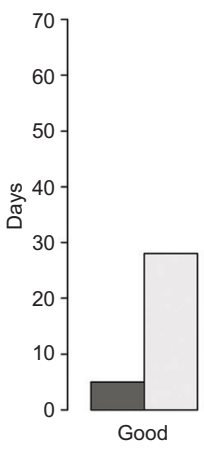

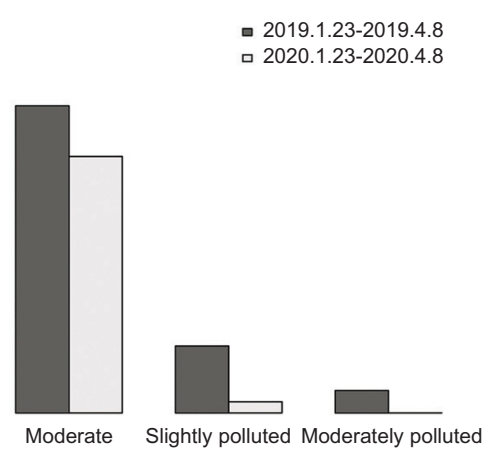

B

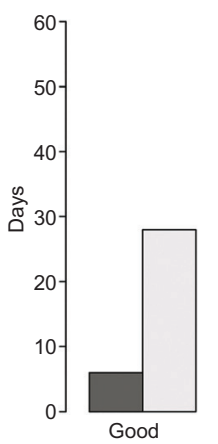

2019.11.7-2020.1.22 口 $2020.1 .23-2020.4 .8$
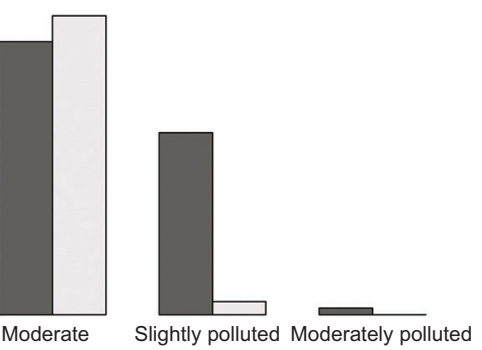

Figure 2 Air quality index comparison of three sections in Wuhan city in China: November 07, 2019-January 22, 2020; January 23, 2020-April 08, 2020 (Total lockdown in Wuhan); and January 23, 2019-April 08, 2019.

in human activity during the COVID-19 outbreak (Figure 1). A similar result was obtained when the calculation was made for Wuhan alone (Figure 2). Based on the data provided by other groups, we think that herein lies the difference between active intervention and passive intervention. The virus caused factories to shut down their production and the transportation dramatically diminished. This is a very passive form of intervention. From the comparative results, we can see that such passive intervention was more effective at improving air quality than active interventions. The Chinese government has implemented active interventions in the past too. We think the situation in China during the COVID-19 outbreak reflects the drop in air pollution throughout the world. It is likely that the improved air quality is due to the suspension of the production and transportation activities. These results are worth thinking about.

In the context of economic globalization, development and balance are critical topics. As a result of the passive intervention of the virus, people stopped the pace of development, leading to a drop in air pollution. The public should take a good look at the COVID-19 epidemic. Humans have a common destiny. We should all join hands and work to improve the environment, modify people's behavior, and avoid nature's vengeance.

\section{Declaration of competing interests}

The authors declare that they have no known competing financial interests or personal relationships that could have appeared to influence the work reported in this paper.

\section{Credit authorship contribution statement}

Zhan-hui Feng: Conceptualization, Methodology, Writing original draft.

Juan Chen: Validation.

Ming-Wei Wang and Wen-tao Gan: Data curation and Writing - review \& editing.

Lan Ye: Data curation, Visualization. 
Yong-ran Cheng and Meng-Yun Zhou: Conceptualization, Supervision.

\section{Acknowledgments}

We are very grateful to the medical staff who are working on the front lines against COVID-19.

\section{References}

1. Urrutia-Pereira M., Mello-da-Silva CA., Solé D. COVID-19 and air pollution: A dangerous association? Allergol Immunopathol (Madr). 2020 Jul 1:S0301-0546(20)30109-9. https://doi. org $/ 10.1016 /$ j.aller.2020.05.004
2. Matthew Warren. United Kingdom unveils ambitious air pollution plan. Science. 2018 Jun 1;360(6392):953. https://doi. org/10.1126/science.360.6392.953

3. The Lancet Planetary Health. The world's biggest threats are environmental risks. Lancet Planet Health. 2018 Feb;2(2):e51. https://doi.org/10.1016/S2542-5196(17)30164-X

4. Hatice Imamoglu. The role of financial sector in energy demand and climate changes: evidence from the developed and developing countries. Environ Sci Pollut Res Int. 2019 Aug;26(22):22794-22811. https://doi.org/10.1007/ s11356-019-05499-y

5. Ahmad S., Ali N., Kausar M., Misbah H., Wahid A. Road toward rapid-molecular point of care test to detect novel SARScoronavirus 2019 (COVID-19): Review from updated literature. Allergol Immunopathol (Madr). 2020 Jul 1:S0301-0546(20)30105-1. https://doi.org/10.1016/j.aller.2020.06.001 